\title{
The first and second expansion of large solutions for quasilinear elliptic equations with weight functions
}

\author{
Yun-Feng Ma ${ }^{1}$ and Zhong Bo Fang ${ }^{2^{*}}$
}

\author{
"Correspondence: \\ fangzb7777@hotmail.com \\ ${ }^{2}$ School of Mathematical Sciences, \\ Ocean University of China, Songling \\ Road 238, Qingdao, 266100, \\ P.R. China \\ Full list of author information is \\ available at the end of the article
}

\begin{abstract}
By the Karamata regular variation theory and comparison principle, we establish the boundary behavior of positive weak solutions for the problem

$$
\Delta_{p} u=b(x) f(u), \quad x \in \Omega,\left.\quad u\right|_{\partial \Omega}=\infty,
$$

where $\Omega$ is a bounded domain with smooth boundary in $R^{N}$, the weight $b(x) \in C^{\alpha}(\bar{\Omega})$, which may be vanishing on the boundary and rapidly varying near the boundary, and the nonlinearity $f$ may be rapidly varying at infinity. For the case $f(s)=s^{m} \pm f_{1}(s)$ with sufficiently large $s$, where $m>p-1$ and $f_{1}$ is normalized regularly varying at infinity with index $m_{1} \in(0, p-1)$, we show the influence of the geometry of $\Omega$ on the boundary behavior of solutions. Finally, we prove the existence and uniqueness of the solution for the problem.
\end{abstract}

MSC: 35B40; 35H30; 35J70; 35J65

Keywords: quasilinear elliptic equation; large solution; blow-up rate; first and second expansion

\section{Introduction and main results}

In this paper, we consider the first and second expansions of positive weak large solutions near the boundary for the quasilinear elliptic problem of the form

$$
\Delta_{p} u=b(x) f(u), \quad x \in \Omega,\left.\quad u\right|_{\partial \Omega}=\infty
$$

where $\Delta_{p} u=\operatorname{div}\left(|\nabla u|^{p-2} \nabla u\right)(p>2), \Omega \subset R^{N}(N \geq 2)$ is a bounded domain with $C^{4}$ smooth boundary, $b(x)$ satisfies $\left(\mathrm{b}_{1}\right)$ and $\left(\mathrm{b}_{2}\right)$, and $f$ satisfies $\left(\mathrm{f}_{1}\right),\left(\mathrm{f}_{2}\right)$, and $\left(\mathrm{f}_{3}\right)$, where

(b $\left.b_{1}\right) b(x) \in C^{\alpha}(\bar{\Omega})$ for some $\alpha \in(0,1)$, and $b(x)$ is positive in $\Omega$;

$\left(\mathrm{b}_{2}\right)$ there exist $k \in \Lambda$ and $b_{0} \in R$ such that

$$
\lim _{d(x) \rightarrow 0} \frac{b(x)}{K^{p-2}(d(x)) k^{p}(d(x))}=b_{0},
$$

(c) $2015 \mathrm{Ma}$ and Fang. This article is distributed under the terms of the Creative Commons Attribution 4.0 International License (http://creativecommons.org/licenses/by/4.0/), which permits unrestricted use, distribution, and reproduction in any medium, provided you give appropriate credit to the original author(s) and the source, provide a link to the Creative Commons license, and indicate if changes were made. 
where $\Lambda$ denotes the set of all positive nondecreasing functions in $C^{1}\left(0, \delta_{0}\right)\left(\delta_{0}>0\right)$ such that

$$
\lim _{t \rightarrow 0+} \frac{d}{d t}\left(\frac{K(t)}{k(t)}\right):=C_{k} \in[0, \infty), \quad K(t)=\int_{0}^{t} k(s) d s
$$

(f $\left.\mathrm{f}_{1}\right) f \in C^{1}[0, \infty), f(0)=0, f$ is increasing in $(0, \infty)$;

(f $\left.f_{2}\right) \int_{1}^{\infty} f^{-\frac{1}{p-1}}(v) d v<\infty$;

$\left(\mathrm{f}_{3}\right)$ there exists $C_{f}>0$ such that $\lim _{s \rightarrow \infty} f^{\prime}(s) \int_{s}^{\infty} \frac{d v}{f(v)}=C_{f}$.

Note that, some basic examples for $k \in \Lambda$ can be given as follows:

(1) $k(t)=t^{\frac{\alpha}{2}}, \alpha>0, C_{k}=2(2+\alpha)^{-1}$;

(2) $k(t)=e^{-t^{-\alpha}}, \alpha>0, C_{k}=0$;

(3) $k(t)=\frac{1}{(-\ln t)^{\alpha}}, \alpha>0, C_{k}=1$;

(4) $k(t)=e^{-e^{t^{-\alpha}}}, \alpha>0, C_{k}=0$;

(5) $k(t)=(\ln (1+t))^{\alpha}, \alpha>0, C_{k}=(1+\alpha)^{-1}$.

$p$-Laplacian equations like (1.1) usually occur in the study of the generalized reactiondiffusion theory, non-Newtonian fluid theory, non-Newtonian filtration, and the turbulent flow of a gas in porous medium. In the non-Newtonian theory, the quantity $p$ is a characteristic of the medium. Media with $p>2$ are called dilatant fluids, and those with $p<2$ are called pseudoplastics. If $p=2$, then they are Newtonian fluids. The $p$-Laplacian operator also appears in the study of torsional creep (elastic for $p=2$ and plastic for $p<2$; see [1]), flow through porous media $\left(p=\frac{3}{2}\right.$; see [2]), and glacial sliding $\left(p \in\left(1, \frac{3}{4}\right]\right.$; see [3]).

We are concerned with the positive weak large solutions for problem (1.1). By a solution for problem (1.1) we understand a function $u \in W_{\mathrm{loc}}^{1, p}(\Omega) \cap L_{\mathrm{loc}}^{\infty}(\Omega)$ that satisfies $\Delta_{p} u=b(x) f(u)$ in the weak sense and $u(x) \rightarrow \infty$ as $d(x)=\operatorname{dist}(x, \partial \Omega) \rightarrow 0$. Sometimes, the solution is also called a large solution, an explosive solution, or a boundary blow-up solution.

The study of large solutions started from the work of Bieberbach [4] for the case $b(x)=1$, $f(u)=e^{u}, p=2$, and $N=2$, which plays an important role in the theories of Riemannian surfaces of negative constant curvatures and automorphic functions. More exactly, if a Riemannian metric of the form $|d s|^{2}=e^{2 u(x)}|d x|^{2}$ has a constant Gaussian curvature $-b^{2}$, then $\Delta u=b^{2} e^{2 u}$. Rademacher [5] extended the results in [4] to the three-dimensional space. Later, $C$ Bandle et al. discussed the existence, uniqueness, and accurate estimate of boundary behavior of large solutions for problem (1.1) with $p=2$ and $b(x), f(u)$ satisfying some proper conditions and obtained some better results dealing with a gradient term (see [4-40] and the references therein). Also, some results in [4-40] have been extended to $p>2$ (see [41-54]). Recently, boundary blow-up problems have been applied to Liouville theorems for logistic-like equations in $R^{N}$ in [13], the analysis of blow-up for a parabolic equation with a nonlinear boundary condition in [14], and the characterization of the long-time behavior of positive solutions for the parabolic equations in $[15,16]$.

However, Cirstea and Rădulescu [17] first introduced Karamata regular variation theory approaching to study the uniqueness and asymptotic behavior of boundary blow-up solutions, which enables us to obtain some qualitative behavior of the boundary blow-up solutions in a general framework. The asymptotic behavior of the boundary blow-up solutions near the boundary has been investigated by many researchers (see [17-39] and their 
references). It is well known that the first-order asymptotic expansion of the solution $u(x)$ in terms of $d(x)$ is independent of the geometry of the domain, whereas the second-order asymptotic expansion of the solution $u(x)$ depends linearly on the mean curvature of the boundary of $\Omega$. There have been many results about the first expansion of large solution for problem (1.1) with $p=2$ and $b(x), f(u)$ satisfying some proper conditions under different regularity boundary conditions (see [21-28]) and the second expansion (see[29-34] and references therein). Bandle and Marcus [29] first studied the influence of the geometry of $\Omega$ on the boundary behavior of the unique radially symmetric solution for problem (1.1) in a ball or an annulus when $f(u)$ is of power form. Their results were extended by Bandle, Anedda, Porru et al. to more general boundary smooth domains, weights, and nonlinearities (see [30-34]). Specially, Cirstea et al. [17, 35-40] used the Karamata regular variation theory, nonlinear transformations, the perturbed method, the upper and lower solution method, and localization method to establish the first and second expansion of large solutions for problem (1.1) with $p=2$, and Cirstea and Rădulescu [17] first introduced the set $\Lambda$. Recently, some results in $[17,35-40]$ have been extended to $p>2$. For instance, Huang et al. [46-48] studied the existence and the first and second expansions of weak solutions when $b(x)$ and $f(u)$ satisfy some suitable conditions, which are different from the conditions in our paper. For more results about the $p$-Laplacian equations, we refer to [49-53] and references therein.

Inspired by the above works, in this paper, we introduce the constants $C_{f}$ and $C_{k}$ to get the asymptotic expansion of solutions for problem (1.1). In particular, when $b(x)=$ $K^{p-2}(d(x)) k(d(x))$ near the boundary and $f(s)=s^{m} \pm f_{1}(s)$ for sufficiently large $s$, where $m>p-1$, and $f_{1}$ satisfies

$\left(f_{4}\right)$ there exists $m_{1} \in(0, p-1)$ such that

$$
\lim _{s \rightarrow \infty} \frac{s f_{1}^{\prime}(s)}{f_{1}(s)}=m_{1}
$$

we show the influence of the geometry of $\Omega$ on the boundary behavior of solutions for problem (1.1). Finally, we prove the existence and uniqueness of the solution for problem (1.1). More precisely, we obtain the following results.

Theorem 1 Letf satisfy $\left(\mathrm{f}_{1}\right)-\left(\mathrm{f}_{3}\right)$, and b satisfy $\left(\mathrm{b}_{1}\right)-\left(\mathrm{b}_{2}\right)$. Suppose that $1<C_{f}<\frac{p-1}{p-2}$ in $\left(\mathrm{f}_{3}\right)$ and

$$
2 C_{f}+C_{k}>2+\frac{p-2}{p-1} C_{f} C_{k} .
$$

Then, for any solution $u$ of problem (1.1), we have

$$
\lim _{d(x) \rightarrow 0} \frac{u(x)}{\phi\left(\gamma K^{2}(d(x))\right)}=1,
$$

where $\phi$ is uniquely defined by

$$
\int_{\phi(t)}^{\infty} f^{-\frac{1}{p-1}}(\mu) d \mu=t, \quad \forall t>0
$$


and

$$
\gamma=\frac{1}{2}\left(\frac{b_{0}}{p-1} \cdot \frac{(p-1)\left(C_{f}-1\right)-C_{f}}{\left(2-C_{k}\right)(p-1)\left(C_{f}-1\right)-C_{f} C_{k}}\right)^{\frac{1}{p-1}} .
$$

Remark 1 By (1.2) one can see that if $C_{f}>1$, then $C_{k}$ can be equal to zero and if $C_{k}>0$, then $C_{f}$ can be equal to 1 .

Theorem 2 Let $f$ satisfy $\left(\mathrm{f}_{1}\right), f(s)=s^{m} \pm f_{1}(s)$ for sufficiently large $s, m>p-1, f_{1}$ satisfy $\left(\mathrm{f}_{4}\right)$, and $b(x)=K^{p-2}(d(x)) k(d(x))$ near the boundary, where $k$ satisfies

$\left(\mathrm{k}_{1}\right) k \in C[0, a] \cap C^{2}(0, a]$ for some $a>0, k(t)>0, k^{\prime}(t)>0, \forall t \in(0, a]$, and $k(0)=0$;

$\left(\mathrm{k}_{2}\right) k \in \Lambda$ with $C_{k}>0$;

$\left(\mathrm{k}_{3}\right) \lim _{t \rightarrow 0} \frac{d^{2}}{d t^{2}}\left(\frac{K(t)}{k(t)}\right)=0$.

The following two results hold:

(1) if $m+1-p>2 m_{1}$, then, in a sufficiently small neighborhood of $\partial \Omega$, for any solution $u$ of problem (1.1), we have

$$
u(x)=C_{1}(K(d(x)))^{-\frac{2(p-1)}{m+1-p}}\left(1+C_{2}(N-1) H(\bar{x}) \frac{K(d(x))}{k(d(x))}+o(d(x))\right)
$$

where

$$
\begin{aligned}
& C_{1}=\left(\left(\frac{2(p-1)}{m+1-p}\right)^{p}\left(p-1+\frac{(m+1-p) C_{k}}{2}\right)\right)^{\frac{1}{m+1-p}}, \\
& C_{2}=\frac{m+1-p}{2(p-1)(m-1)+(m+1)(m+1-p) C_{k}} .
\end{aligned}
$$

(2) if $m+1-p \leq 2 m_{1}$ and $k(t)=t^{\frac{\theta}{2}}$ with $\theta>0$ such that $\frac{\theta}{2+\theta}>\frac{2 m_{1}-(m+p-1)}{m+1-p}$, then (1) still holds.

Remark 2 Some basic examples of $k$ that satisfy $\left(\mathrm{k}_{1}\right)-\left(\mathrm{k}_{3}\right)$ can be given as follows:

(1) $k(t)=t^{\frac{\alpha}{2}}, \alpha>0$, where $C_{k}=\frac{2}{2+\alpha}$;

(2) $k(t)=e^{t^{\alpha}}-1, \alpha>1$, where $C_{k}=\frac{1}{1+\alpha}$;

(3) $k(t)=\ln \left(1+t^{\alpha}\right), \alpha>1$, where $C_{k}=\frac{1}{1+\alpha}$.

Remark 3 If $k(t)=(\ln (1+t))^{\alpha}, \alpha>0$, then $\lim _{t \rightarrow 0+} \frac{d^{2}}{d t^{2}}\left(\frac{K(t)}{k(t)}\right)=\frac{\alpha}{2(1+\alpha)(2+\alpha)}$. In this case, $k(t)$ does not satisfy $\left(\mathrm{k}_{3}\right)$.

This paper is organized as follows. In Section 2, we present some notation and results in regular variation theory. Theorems 1 and 2 will be proved in Section 3. Finally, we prove the existence and uniqueness of the solution for problem (1.1) in Appendices A.1 and A.2.

\section{Preliminary results}

\subsection{Properties of regularly varying function}

Karamata regular variation theory was established by Karamata in 1930 and is a basic tool in stochastic process. In 1970, Haan improved the results, which have been applied in 
stochastic process, analytical function theory, integral functions, integral transform and asymptotic estimation of an integral sequence (see [55-57]).

In this section, we recall some basic definitions and qualities in regular variation theory.

Definition 1 A positive measurable function $f$ defined on $[a, \infty)$ for some $a>0$ is called regularly varying at infinity with index $\rho$ (written as $f \in R V_{\rho}$ ) if for each $\xi>0$ and some $\rho \in R$

$$
\lim _{t \rightarrow \infty} \frac{f(\xi t)}{f(t)}=\xi^{\rho}
$$

In particular, when $\rho=0, f$ is called slowly varying at infinity. Clearly, if $f \in R V_{\rho}$, then $L(s):=\frac{f(s)}{s^{\rho}}$ is slowly varying at infinity.

Definition 2 A positive measurable function $f$ defined on $[a, \infty)$ for some $a>0$ is called rapidly varying at infinity if for each $\rho>1$,

$$
\lim _{s \rightarrow \infty} \frac{f(s)}{s^{\rho}}=\infty .
$$

Some basic examples of slowly varying functions at infinity are listed as follows:

(1) every measurable function on $[a, \infty)$ which has a positive limit at infinity;

(2) $(\ln t)^{s}$ and $(\ln (\ln t))^{s}, s \in R$;

(3) $e^{(\ln t)^{s}}, 0<s<1$.

Some basic examples of rapidly varying functions at infinity are given as follows:

(1) $e^{t}$ and $e^{e^{t}}$;

(2) $e^{e^{(\ln t)^{s}}}, e^{e^{t^{s}}}$ and $e^{e^{t^{s}}}, s>0$;

(3) $t^{\gamma} e^{(\ln t)^{q}}$ and $(\ln t)^{\gamma} e^{(\ln t)^{q}}, q>1, \gamma \in R$;

(4) $(\ln t)^{\gamma} e^{t^{q}}$ and $t^{\gamma} e^{t^{\gamma}}, q>0, \gamma \in R$.

We see that a positive measurable function $h$ defined on $(0, a)$ for some $a>0$ is regularly varying at zero with index $\sigma$ (written as $g \in R V Z_{\sigma}$ ) if $t \rightarrow g\left(\frac{1}{t}\right)$ belongs to $R V_{-\sigma}$. Similarly, $g$ is called rapidly varying at zero if $t \rightarrow g\left(\frac{1}{t}\right)$ is rapidly varying at infinity.

Proposition 1 (Uniform convergence theorem) If $\in R V_{\rho}$, then (2.1) holds uniformly for $\xi \in\left[c_{1}, c_{2}\right]$ with $0<c_{1}<c_{2}$. Moreover, if $\rho<0$, then uniform convergence holds on intervals of the form $\left(a_{1}, \infty\right)$ with $a_{1}>0$; if $\rho>0$, then uniform convergence holds on intervals $\left(0, a_{1}\right]$, provided that $f$ is bounded on $\left(0, a_{1}\right]$ for all $a_{1}>0$.

Proposition 2 (Representation theorem) A function $L$ is slowly varying at infinity if and only if it can be written in the form

$$
L(s)=\varphi(s) \exp \left(\int_{a_{1}}^{s} \frac{y(t)}{t} d t\right), \quad s \geq a_{1}
$$

for some $a_{1} \geq a$, where the functions $\varphi$ and $y$ are measurable and as $s \rightarrow \infty, y(s) \rightarrow 0$ and $\varphi(s) \rightarrow c_{0}>0$.

We say that

$$
\hat{L}(s)=c_{0} \exp \left(\int_{a_{1}}^{s} \frac{y(t)}{t} d t\right), \quad s \geq a_{1},
$$


is normalized slowly varying at infinity and

$$
f(s)=c_{0} s^{\rho} \hat{L}(s), \quad s \geq a_{1},
$$

is normalized regularly varying at infinity with index $\rho$ (written as $\left.f \in N R V_{\rho}\right)$.

Similarly, $g$ is called normalized regularly varying at zero with index $\sigma$ (written as $g \in$ $\left.N R V Z_{\sigma}\right)$ if $t \rightarrow g\left(\frac{1}{t}\right)$ belongs to $N R V_{-\sigma}$. A function $f \in R V_{\rho}$ belongs to $N R V_{\rho}$ if and only if

$$
f \in C^{1}\left[a_{1}, \infty\right) \text { for some } a_{1}>0 \text { and } \lim _{s \rightarrow \infty} \frac{s f^{\prime}(s)}{f(s)}=\rho .
$$

Proposition 3 If functions $L_{1}, L_{2}$ are slowly varying at infinity, then

(1) $L^{\sigma}$ for every $\sigma \in R, c_{1} L+c_{2} L_{1}\left(c_{1} \geq 0, c_{2} \geq 0\right.$ with $\left.c_{1}+c_{2}>0\right), L \circ L_{1}\left(\right.$ if $L_{1}(t) \rightarrow+\infty$ as $t \rightarrow+\infty)$ are also slowly varying at infinity;

(2) for every $\theta>0, t^{\theta} L(t) \rightarrow+\infty$ and $t^{-\theta} L(t) \rightarrow 0$ as $t \rightarrow \infty$;

(3) for $\rho \in R, \frac{\ln (L(t))}{\ln t} \rightarrow 0$ and $\frac{\ln \left(t^{\rho} L(t)\right)}{\ln t} \rightarrow \rho$ as $t \rightarrow+\infty$.

Proposition 4 If $f_{1} \in R V_{\rho_{1}}, f_{2} \in R V_{\rho_{2}}$ with $\lim _{t \rightarrow \infty} f_{2}(t)=\infty$, then $f_{1} \circ f_{2} \in R V_{\rho_{1} \rho_{2}}$.

Proposition 5 (Asymptotic behavior) If a function $L$ is slowly varying at infinity, then for $a \geq 0$ and $t \rightarrow \infty$, we have

(1) $\int_{a}^{t} s^{\beta} L(s) d s \cong(\beta+1)^{-1} t^{1+\beta} L(t)$ for $\beta>-1$;

(2) $\int_{t}^{\infty} s^{\beta} L(s) d s \cong(-\beta-1)^{-1} t^{1+\beta} L(t)$ for $\beta<-1$.

Proposition 6 (Asymptotic behavior) If a function $H$ is slowly varying at infinity, then for $a>0$ and $t \rightarrow 0^{+}$, we have

(1) $\int_{0}^{t} s^{\beta} H(s) d s \cong(\beta+1)^{-1} t^{1+\beta} H(t)$ for $\beta>-1$;

(2) $\int_{t}^{\infty} s^{\beta} H(s) d s \cong(-\beta-1)^{-1} t^{1+\beta} H(t)$ for $\beta<-1$.

\subsection{Auxiliary results}

In this section, we give some auxiliary results, which will be used in Theorems 1 and 2 .

Lemma $1[38,40]$

(I) If $k \in \Lambda$, then we have:

(1) $\lim _{t \rightarrow 0+} \frac{K(t)}{k(t)}=0$;

(2) $C_{k} \in[0,1]$ and $\lim _{t \rightarrow 0+} \frac{K(t) k^{\prime}(t)}{k^{2}(t)}=1-C_{k}$.

(II) $\left(\mathrm{k}_{1}\right)-\left(\mathrm{k}_{3}\right)$ implies that

(3) $\lim _{t \rightarrow 0+}\left(\frac{K(t) k^{\prime}(t)}{k^{2}(t)}-\left(1-C_{k}\right)\right) \frac{k(t)}{K(t)}=0$.

Lemma 2 [40] Let $f$ satisfy $\left(\mathrm{f}_{1}\right),\left(\mathrm{f}_{2}\right)$, and $\left(\mathrm{f}_{3}\right)$. Then

(1) $C_{f} \in[1, \infty)$;

(2) there exists $S_{0}>0$ such that $\frac{f(s)}{s^{q}}$ is increasing in $\left[S_{0}, \infty\right)$, where $q \in\left(p-1, \frac{C_{f}}{C_{f}-1}\right)$ for $1<C_{f}<\frac{p-1}{p-2}$ and $q \in(p-1, \infty)$ for $C_{f}=1$;

(3) $f$ satisfies the Keller-Osserman condition

$$
\int_{1}^{\infty} \frac{d t}{\left(p^{\prime} F(t)\right)^{\frac{1}{p}}}<\infty, \quad F(t)=\int_{0}^{t} f(s) d s
$$

where $\frac{1}{p^{\prime}}+\frac{1}{p}=1$; 
(4) $\left(\mathrm{f}_{3}\right)$ holds for $C_{f}>1$ if and only iff $\in N R V \frac{C_{f}}{C_{f^{-1}}}$;

(5) $C_{f}=1$, and $f$ is rapidly varying at infinity.

Lemma 3 Let $f$ satisfy $\left(\mathrm{f}_{1}\right)-\left(\mathrm{f}_{3}\right)$, and $\phi$ be the solution for the problem

$$
\int_{\phi(t)}^{\infty} f^{-\frac{1}{p-1}}(s) d s=t, \quad \forall t>0 .
$$

\section{Then we have}

(1) $-\phi^{\prime}(t)=f^{\frac{1}{p-1}}(\phi(t)), \phi(t)>0, t>0, \phi(0):=\lim _{t \rightarrow 0+} \phi(t)=+\infty$, $\phi^{\prime \prime}(t)=\frac{1}{p-1} f^{\frac{2}{p-1}-1}(\phi(t)) f^{\prime}(\phi(t)) ;$

(2) $\lim _{t \rightarrow 0+} \frac{t \phi^{\prime}(t)}{\phi(t)}=-\frac{(p-1)\left(C_{f}-1\right)}{C_{f}-(p-1)\left(C_{f}-1\right)}$, i.e., $\phi \in N R V Z_{-\frac{(p-1)\left(C_{f}-1\right)}{C_{f}(p-1)\left(C_{f}-1\right)}}$;

(3) $\lim _{t \rightarrow 0+} \frac{t \phi^{\prime \prime}(t)}{\phi^{\prime}(t)}=-\frac{C_{f}}{C_{f}-(p-1)\left(C_{f}-1\right)}$, i.e., $-\phi^{\prime} \in N R V Z_{-\frac{C_{f}}{C_{f}(p-1)\left(C_{f}-1\right)}}$;

(4) $\lim _{t \rightarrow 0+} \frac{\ln (\phi(t))}{-\ln t}=\frac{(p-1)\left(C_{f}-1\right)}{C_{f}-(p-1)\left(C_{f}-1\right)}, \lim _{t \rightarrow 0+} \frac{\ln \left(\phi^{\prime}(t)\right)}{-\ln t}=\frac{(p-1) C_{f}}{C_{f}-(p-1)\left(C_{f}-1\right)}$.

Proof (1) By the definition of $\phi$ and a direct calculation we can show (1).

(2) It follows from Proposition 5 that

$$
\begin{aligned}
\lim _{t \rightarrow 0+} \frac{t \phi^{\prime}(t)}{\phi(t)}= & -\lim _{t \rightarrow 0+} \frac{t f^{\frac{1}{p-1}}(\phi(t))}{\phi(t)} \\
= & -\lim _{u \rightarrow \infty} \frac{(f(u))^{\frac{1}{p-1}}}{u} \int_{u}^{\infty} \frac{d v}{f^{\frac{1}{p-1}(v)}} \\
= & -\lim _{u \rightarrow \infty} u^{\frac{C_{f}}{(p-1)\left(C_{f}-1\right)}-1} \hat{L}^{\frac{1}{p-1}}(u) \int_{u}^{\infty} v^{-\frac{C_{f}}{(p-1)\left(C_{f}-1\right)}} \hat{L}^{-\frac{1}{p-1}}(v) d v \\
= & -\left(\frac{C_{f}}{(p-1)\left(C_{f}-1\right)}-1\right)^{-1} \\
& \cdot \lim _{u \rightarrow \infty} u^{\frac{C_{f}}{(p-1)\left(C_{f}-1\right)}-1} \hat{L}^{\frac{1}{p-1}}(u) u^{-\frac{C_{f}}{(p-1)\left(C_{f}-1\right)}+1} \hat{L}^{-\frac{1}{p-1}}(u) \\
= & -\frac{(p-1)\left(C_{f}-1\right)}{C_{f}-(p-1)\left(C_{f}-1\right)} .
\end{aligned}
$$

(3) $\left(f_{3}\right)$ implies that

$$
\begin{aligned}
\lim _{t \rightarrow 0+} \frac{t \phi^{\prime \prime}(t)}{\phi^{\prime}(t)} & =-\frac{1}{p-1} \lim _{t \rightarrow 0+} t f^{\frac{1}{p-1}-1}(\phi(t)) f^{\prime}(\phi(t)) \\
& =-\frac{1}{p-1} \cdot \lim _{u \rightarrow \infty} f^{\frac{1}{p-1}-1}(u) f^{\prime}(u) \int_{u}^{\infty} \frac{d v}{f^{\frac{1}{p-1}}(v)} \\
& =-\frac{1}{p-1} \cdot \lim _{u \rightarrow \infty} \frac{u f^{\prime}(u) f^{\frac{1}{p-1}}(u)}{u(u)} \int_{u}^{\infty} \frac{d v}{f^{\frac{1}{p-1}}(v)} \\
& =-\frac{1}{p-1} \cdot \frac{(p-1)\left(C_{f}-1\right)}{C_{f}-(p-1)\left(C_{f}-1\right)} \cdot \frac{C_{f}}{C_{f}-1} \\
& =-\frac{C_{f}}{C_{f}-(p-1)\left(C_{f}-1\right)} .
\end{aligned}
$$

The last result (4) follows from (2)-(3) and Proposition 3(3). 
Lemma 4 Under the hypotheses in Theorem 2 , let $k \in \Lambda$ and

$$
\Phi(t)=(K(t))^{-\frac{2(p-1)}{m+1-p}}(1+h(t))
$$

with $\lim _{t \rightarrow 0} h(t)=0$. The following two results hold:

(1) if $m+1-p>2 m_{1}$, then $k(t)(K(t))^{\frac{(m+p-1)(p-1)}{m+1-p}} f_{1}(\Phi(t)) \rightarrow 0$ as $t \rightarrow 0$;

(2) if $m+1-p \leq 2 m_{1}$ and $k(t)=t^{\frac{\theta}{2}}$ with $\theta>0$ such that $\frac{\theta}{2+\theta}>\frac{2 m_{1}-(m+p-1)}{m+1-p}$, then (1) still holds.

Proof We know that $f_{1} \in N R V_{m_{1}}$ by $\left(\mathrm{f}_{4}\right)$ with $m_{1} \in(0, m)$ and $f_{1}(s)=c_{0} s^{m_{1}} \hat{L}(s)$ for sufficiently large $s$, where $\hat{L}$ is normalized slowly varying at infinity, and $c_{0}>0$.

Let

$$
\Phi_{1}(t)=(K(t))^{-\frac{2(p-1)}{m+1-p}} .
$$

We see that $\hat{L}\left(\Phi_{1}(t)\right)$ is also normalized slowly varying at zero, and by a similar argument as in Propositions 1 and 3(2), for every $\beta>0$ and $t \rightarrow 0+$, we have

$$
\left(\Phi_{1}(t)\right)^{\beta} \hat{L}\left(\Phi_{1}(t)\right) \rightarrow 0 \quad \text { and } \quad \hat{L}(\Phi(t))\left(\hat{L}\left(\Phi_{1}(t)\right)\right)^{-1}(1+h(t))^{m_{1}} \rightarrow 0 .
$$

(1) If $m+1-p>2 m_{1}$, let $2 \beta \in\left(0, m+p-1-2 m_{1}\right)$, then by (2.8) we have

$$
\begin{aligned}
& k(t)(K(t))^{\frac{(m+p-1)(p-1)}{m+1-p}} f_{1}(\Phi(t)) \\
&= k(t)(K(t))^{\frac{(m+p-1)(p-1)}{m+1-p}} c_{0}(\Phi(t))^{m_{1}} \hat{L}(\Phi(t)) \\
&= c_{0} k(t)(K(t))^{\frac{(m+p-1)(p-1)-2(p-1) m_{1}-2(p-1) \beta}{m+1-p}}\left(\Phi_{1}(t)\right)^{\beta} \hat{L}\left(\Phi_{1}(t)\right) \\
& \cdot \hat{L}(\Phi(t))\left(\hat{L}\left(\Phi_{1}(t)\right)\right)^{-1}(1+h(t))^{m_{1}} \rightarrow 0 \quad \text { as } t \rightarrow 0 .
\end{aligned}
$$

(2) If $m+1-p \leq 2 m_{1}$ and $k(t)=t^{\frac{\theta}{2}}$ with $\theta>0$ such that $\frac{\theta}{2+\theta}>\frac{2 m_{1}-(m+p-1)}{m+1-p}$ and $\frac{2(p-1) m_{1}}{m+1-p} \in$ $\left(0, \frac{\theta}{2+\theta}-\frac{2 m_{1}-(m+p-1)}{m+1-p}\right)$, then we get

$$
\begin{aligned}
& k(t)(K(t))^{\frac{(m+p-1)(p-1)}{m+1-p}} f_{1}(\Phi(t)) \\
& \quad=c t^{\sigma}\left(\Phi_{1}(t)\right)^{\beta} \hat{L}\left(\Phi_{1}(t)\right) \hat{L}(\Phi(t))\left(\hat{L}\left(\Phi_{1}(t)\right)\right)^{-1}(1+h(t))^{m_{1}} \rightarrow 0 \quad \text { as } t \rightarrow 0,
\end{aligned}
$$

where

$$
\begin{aligned}
& c=c_{0}\left(\frac{\theta+2}{2}\right)^{\frac{(p-1)\left(2 m_{1}+2 \beta-m+1-p\right)}{m+1-p}}, \\
& \sigma=\frac{\theta(m+1-p)-(2+\theta)(p-1)\left(2 m_{1}+2 \beta-m+1-p\right)}{2(m+1-p)}>0 .
\end{aligned}
$$

\section{Proofs of main results}

In this section, we mainly prove Theorem 1 and Theorem 2. For the proofs, we use the upper and lower solution method. One critical step is to set up the comparison principle. Thus, we first give the comparison principle in general form for quasilinear elliptic equations. 
Lemma 5 [54] Suppose that $D$ is a bounded domain in $R^{N}$ and $a(x)$ and $\beta(x)$ are continuous functions on $D$ with $\|a\|_{L^{\infty}(D)}<\infty, \beta(x) \geq 0, \beta(x) \neq 0$ for $x \in D$. Let $u_{1}, u_{2} \in C^{1}(D)$ be positive in $D$ and satisfy in the sense of distributions

$$
\begin{aligned}
& -\Delta_{p} u_{1}-a(x) u_{1}^{p-1}+\beta(x) g\left(u_{1}\right) \geq 0 \geq-\Delta_{p} u_{2}-a(x) u_{2}^{p-1}+\beta(x) g\left(u_{2}\right), \\
& \varlimsup_{d(x, \partial \Omega) \rightarrow 0}\left(u_{2}^{p-1}-u_{1}^{p-1}\right) \leq 0,
\end{aligned}
$$

where $g \in C^{0}([0, \infty))$, and $\frac{g(s)}{s^{p-1}}$ is increasing for

$$
s \in\left(\inf _{D}\left\{u_{1}, u_{2}\right\}, \sup _{D}\left\{u_{1}, u_{2}\right\}\right) .
$$

Then $u_{1} \geq u_{2}$ in $D$.

Next, fix $\epsilon>0$. For all $\delta>0$, we define $\Omega_{\delta}=\{x \in \Omega: 0<d(x)<\delta\}$. Since $\Omega$ is smooth, there exists $\delta_{0}>0$ such that $d \in C^{2}\left(\Omega_{\delta_{0}}\right)$ and

$$
|\nabla d(x)|=1, \quad \Delta d(x)=-(N-1) H(\bar{x}) d(x)+o(1), \quad \forall x \in \Omega_{\delta_{0}} .
$$

Proof of Theorem 1 Let $\epsilon \in\left(0, \frac{b_{0}}{4}\right)$ and $\gamma_{1}=\gamma-\frac{2 \epsilon \gamma}{b_{0}}, \gamma_{2}=\gamma+\frac{2 \epsilon \gamma}{b_{0}}$. We have

$$
\frac{\gamma}{2}<\gamma_{1}<\gamma<\gamma_{2}<2 \gamma
$$

Set

$$
\begin{aligned}
& d_{1}(x)=d(x)-\rho, \quad d_{2}(x)=d(x)+\rho, \\
& \bar{u}_{\epsilon}=\phi\left(\gamma_{1} K^{2}\left(d_{1}(x)\right)\right), \quad x \in D_{\rho}^{-} \quad \text { and } \quad \underline{u}_{\epsilon}=\phi\left(\gamma_{2} K^{2}\left(d_{2}(x)\right)\right), \quad x \in D_{\rho}^{+} .
\end{aligned}
$$

Then, for $x \in D_{\rho}^{-}$,

$$
\begin{aligned}
\Delta_{p} \bar{u}_{\epsilon} & -b(x) f\left(\bar{u}_{\epsilon}\right) \\
= & (p-1)\left|2 \gamma_{1} \phi^{\prime}\left(\gamma_{1} K^{2}\left(d_{1}(x)\right)\right) K\left(d_{1}(x)\right) k\left(d_{1}(x)\right)\right|^{p-2} \\
& \cdot\left(\phi^{\prime \prime}\left(\gamma_{1} K^{2}\left(d_{1}(x)\right)\right) 4 \gamma_{1}^{2} K^{2}\left(d_{1}(x)\right) k^{2}\left(d_{1}(x)\right)\right. \\
& +\phi^{\prime}\left(\gamma_{1} K^{2}\left(d_{1}(x)\right)\right) 2 \gamma_{1} k^{2}\left(d_{1}(x)\right)+\phi^{\prime}\left(\gamma_{1} K^{2}\left(d_{1}(x)\right)\right) 2 \gamma_{1} K\left(d_{1}(x)\right) k^{\prime}\left(d_{1}(x)\right) \\
& \left.+2 \gamma_{1} \phi^{\prime}\left(\gamma_{1} K^{2}\left(d_{1}(x)\right)\right) K\left(d_{1}(x)\right) k\left(d_{1}(x)\right) \Delta d(x)\right) \\
& -b_{0} K^{p-2}\left(d_{1}(x)\right) k^{p}\left(d_{1}(x)\right) f\left(\phi\left(\gamma_{1} K^{2}\left(d_{1}(x)\right)\right)\right) \\
= & (p-1)\left(2 \gamma_{1}\right)^{p-2} f\left(\phi\left(\gamma_{1} K^{2}\left(d_{1}(x)\right)\right)\right) K^{p-2}\left(d_{1}(x)\right) k^{p}\left(d_{1}(x)\right) \\
& \cdot\left(4 \gamma_{1}\left(\frac{\gamma_{1} K^{2}\left(d_{1}(x)\right) \phi^{\prime \prime}\left(\gamma_{1} K^{2}\left(d_{1}(x)\right)\right)}{-\phi^{\prime}\left(\gamma_{1} K^{2}\left(d_{1}(x)\right)\right)}-\frac{C_{f}}{C_{f}-(p-1)\left(C_{f}-1\right)}\right)\right. \\
& -2 \gamma_{1}\left(\frac{K\left(d_{1}(x)\right) k^{\prime}\left(d_{1}(x)\right)}{k^{2}\left(d_{1}(x)\right)}-\left(1-C_{k}\right)\right)-2 \gamma_{1} \frac{K\left(d_{1}(x)\right)}{k\left(d_{1}(x)\right)} \Delta d(x) \\
& -\left(\frac{1}{\left(2 \gamma_{1}\right)^{p-2}(p-1)} \frac{b(x)}{K^{p-2}\left(d_{1}(x)\right) k^{p}\left(d_{1}(x)\right)}-\frac{b_{0}}{\left(2 \gamma_{1}\right)^{p-2}(p-1)}\right) \\
& \left.-\frac{b_{0}}{\left(2 \gamma_{1}\right)^{p-2}(p-1)}+\frac{4 \gamma_{1} C_{f}}{C_{f}-(p-1)\left(C_{f}-1\right)}-2 \gamma_{1}\left(1-C_{k}\right)-2 \gamma_{1}\right) .
\end{aligned}
$$


By $\left(b_{1}\right),\left(b_{2}\right)$, and Lemmas 1-3 we see that there exists $\delta_{\epsilon} \in\left(0, \frac{\delta_{0}}{2}\right)$ sufficiently small such that

$\left(\mathrm{r}_{1}\right)\left(b_{0}-\epsilon\right) K^{p-2}(d(x)-\rho) k^{p}(d(x)-\rho) \leq\left(b_{0}-\epsilon\right) K^{p-2}(d(x)) k^{p}(d(x))<b(x), x \in D_{\rho}^{-}=$ $\Omega_{2 \delta_{\epsilon}} / \bar{\Omega}_{\rho} ; b(x)<\left(b_{0}+\epsilon\right) K^{p-2}(d(x)) k^{p}(d(x)) \leq\left(b_{0}+\epsilon\right) K^{p-2}(d(x)+\rho) k^{p}(d(x)+\rho), x \in$ $D_{\rho}^{+}=\Omega_{2 \delta_{\epsilon}-\rho}$, where $\rho \in\left(0, \delta_{\epsilon}\right)$,

$\left(\mathrm{r}_{2}\right)$

$$
\begin{aligned}
& 4 \gamma\left|\gamma_{1} \frac{K^{2}(t) \phi^{\prime \prime}\left(\gamma_{1} K^{2}(t)\right)}{\phi^{\prime}\left(\gamma_{1} K^{2}(t)\right)}-\frac{C_{f}}{C_{f}-(p-1)\left(C_{f}-1\right)}\right|+2 \gamma\left|\frac{K(t) k^{\prime}(t)}{k^{2}(t)}-\left(1-C_{k}\right)\right| \\
& \quad+2 \gamma \frac{K(t)}{k(t)}|\Delta d(x)|<\epsilon, \quad \forall(x, t) \in \Omega_{2 \delta_{\epsilon}} \times\left(0,2 \delta_{\epsilon}\right),
\end{aligned}
$$

and by the value of $\gamma_{1}$ in Theorem 1 ,

$$
-\frac{b_{0}}{\left(2 \gamma_{1}\right)^{p-2}(p-1)}+\frac{4 \gamma_{1} C_{f}}{C_{f}-(p-1)\left(C_{f}-1\right)}-2 \gamma_{1}\left(1-C_{k}\right)-2 \gamma_{1}=0 .
$$

Then

$$
\Delta_{p} \bar{u}_{\epsilon}-b(x) f\left(\bar{u}_{\epsilon}\right) \leq 0,
$$

i.e., $\bar{u}_{\epsilon}$ is a supersolution of Eq. (1.1) in $D_{\rho}^{-}$.

Similarly, we can show that $\underline{u}_{\epsilon}$ is a subsolution of Eq. (1.1) in $D_{\rho}^{+}$.

Now let $u$ be an arbitrary solution of problem (1.1) and $C_{1}\left(\delta_{\epsilon}\right):=\max _{d(x) \geq \delta_{\epsilon}} u(x)$. We see that

$$
u \leq C_{1}\left(\delta_{\epsilon}\right)+\bar{u}_{\epsilon} \quad \text { on } \partial D_{\rho}^{-} .
$$

Since $\phi$ is decreasing and $\gamma_{1}>\gamma$, we have

$$
\underline{u}_{\epsilon} \leq \phi\left(\gamma K\left(2 \delta_{\epsilon}\right)\right):=C_{2}\left(\delta_{\epsilon}\right) \quad \text { whenever } d(x)=2 \delta_{\epsilon}-\rho
$$

and

$$
\underline{u}_{\epsilon} \leq u+C_{2}\left(\delta_{\epsilon}\right) \text { on } \partial D_{\rho}^{+}
$$

It follows by $\left(f_{1}\right)$ and Lemma 5 that

$$
u \leq C_{1}\left(\delta_{\epsilon}\right)+\bar{u}_{\epsilon}, \quad x \in D_{\rho}^{-} \quad \text { and } \quad \underline{u}_{\epsilon} \leq u+C_{2}\left(\delta_{\epsilon}\right), \quad x \in D_{\rho}^{+} .
$$

Hence, letting $\rho \rightarrow 0$, we have, for $x \in D_{\rho}^{-} \cap D_{\rho}^{+}$,

$$
1-\frac{C_{2}\left(\delta_{\epsilon}\right)}{\phi\left(\gamma_{2} K^{2}(d(x))\right)} \leq \frac{u(x)}{\phi\left(\gamma_{2} K^{2}(d(x))\right)}
$$

and

$$
\frac{u(x)}{\phi\left(\gamma_{1} K^{2}(d(x))\right)} \leq 1+\frac{C_{1}\left(\delta_{\epsilon}\right)}{\phi\left(\gamma_{1}\right) K^{2}(d(x))} .
$$


Consequently,

$$
1 \leq \liminf _{d(x) \rightarrow 0} \frac{u(x)}{\phi\left(\gamma_{2} K^{2}(d(x))\right)} \quad \text { and } \quad \limsup _{d(x) \rightarrow 0} \frac{u(x)}{\phi\left(\gamma_{1} K^{2}(d(x))\right)} \leq 1 .
$$

Thus, letting $\epsilon \rightarrow 0$, we have

$$
1 \leq \liminf _{d(x) \rightarrow 0} \frac{u(x)}{\phi\left(\gamma K^{2}(d(x))\right)} \quad \text { and } \quad \limsup _{d(x) \rightarrow 0} \frac{u(x)}{\phi\left(\gamma K^{2}(d(x))\right)} \leq 1,
$$

that is,

$$
\lim _{d(x) \rightarrow 0} \frac{u(x)}{\phi\left(\gamma K^{2}(d(x))\right)}=1 .
$$

The proof is complete.

Proof of Theorem 2 Let $\epsilon \in(0,1)$ and

$$
\begin{array}{ll}
\bar{u}_{\epsilon}=C_{1}\left(K\left(d_{1}(x)\right)\right)^{-\frac{2(p-1)}{m+1-p}}\left(1+C_{2}(N-1)(H(\bar{x})+\epsilon) \frac{K\left(d_{1}(x)\right)}{k\left(d_{1}(x)\right)}\right), & x \in D_{\rho}^{-}, \\
\underline{u}_{\epsilon}=C_{1}\left(K\left(d_{1}(x)\right)\right)^{-\frac{2(p-1)}{m+1-p}}\left(1+C_{2}(N-1)(H(\bar{x})-\epsilon) \frac{K\left(d_{2}(x)\right)}{k\left(d_{2}(x)\right)}\right), \quad x \in D_{\rho}^{+} .
\end{array}
$$

Using Lemma 4 and a direct calculation we see that, for $x \in D_{\rho}^{-}$,

$$
\begin{aligned}
& K^{p-2}\left(d_{1}(x)\right) k^{p}\left(d_{1}(x)\right) f\left(\bar{u}_{\epsilon}(x)\right) \\
&=K^{p-2}\left(d_{1}(x)\right) k^{p}\left(d_{1}(x)\right)\left(\bar{u}_{\epsilon}^{m}(x) \pm c_{0} \bar{u}_{\epsilon}^{m_{1}}(x) \hat{L}\left(\bar{u}_{\epsilon}(x)\right)\right) \\
&=K^{p-2}\left(d_{1}(x)\right) k^{p}\left(d_{1}(x)\right) \\
& \cdot\left[C_{1}^{m}\left(K\left(d_{1}(x)\right)\right)^{-\frac{2 m(p-1)}{m+1-p}}\left(1+m C_{2}(N-1)(H(\bar{x})+\epsilon) \frac{K\left(d_{1}(x)\right)}{k\left(d_{1}(x)\right)}\right)\right. \\
& \pm c_{0} C_{1}^{m_{1}}\left(K\left(d_{1}(x)\right)\right)^{-\frac{2 m_{1}(p-1)}{m+1-p}} \hat{L}\left(\bar{u}_{\epsilon}(x)\right) \\
&\left.\cdot\left(1+C_{2}(N-1)(H(\bar{x})+\epsilon) \frac{K\left(d_{1}(x)\right)}{k\left(d_{1}(x)\right)}\right)^{m_{1}}\right] \\
&=K^{p-2}\left(d_{1}(x)\right) k^{p-1}\left(d_{1}(x)\right)\left(K\left(d_{1}(x)\right)\right)^{-\frac{2 m(p-1)}{m+1-p}+1} \\
& \quad \cdot\left[C_{1}^{m} \frac{k\left(d_{1}(x)\right)}{K\left(d_{1}(x)\right)}+m C_{1}^{m} C_{2}(N-1)(H(\bar{x})+\epsilon)\right. \\
& \pm\left(K\left(d_{1}(x)\right)\right)^{-\frac{2\left(m_{1}+m\right)(p-1)}{m+1-p}-1} k(t) \hat{L}\left(\bar{u}_{\epsilon}(x)\right) \\
&\left.\cdot\left(1+C_{2}(N-1)(H(\bar{x})+\epsilon) \frac{K\left(d_{1}(x)\right)}{k\left(d_{1}(x)\right)}\right)^{m_{1}}\right]
\end{aligned}
$$

and

$$
\begin{aligned}
\left|\nabla \bar{u}_{\epsilon}\right|= & \left(K\left(d_{1}(x)\right)\right)^{-\frac{m+p-1}{m+1-p}} k\left(d_{1}(x)\right) \nabla d(x) \\
& \cdot\left(1+C_{2}(N-1)(H \bar{x}+\epsilon) \frac{K\left(d_{1}(x)\right)}{k\left(d_{1}(x)\right)}\right)
\end{aligned}
$$




$$
\begin{aligned}
& +\left.C_{1}\left(K\left(d_{1}(x)\right)\right)^{-\frac{2(p-1)}{m+1-p}} C_{2}(N-1)(H(\bar{x}+\epsilon)) \frac{d}{d t}\left(\frac{K(t)}{k(t)}\right)\right|_{t=d_{1}(x)} \nabla d(x), \\
& \Delta_{p} \bar{u}_{\epsilon}=(p-1)\left|\nabla \bar{u}_{\epsilon}\right|^{p-2} \times\left[\frac{2(p-1)(m+p-1)}{(m+1-p)^{2}}\right. \\
& \cdot\left(K\left(d_{1}(x)\right)\right)^{-\frac{m+p-1}{m+1-p}-1} k^{2}\left(d_{1}(x)\right)\left(1+C_{2}(N-1)(H(\bar{x})+\epsilon) \frac{K\left(d_{1}(x)\right)}{k\left(d_{1}(x)\right)}\right) \\
& -\frac{2(p-1) C_{1}}{(m+1-p)}\left(K\left(d_{1}(x)\right)\right)^{-\frac{m+p-1}{m+1-p}} k^{\prime}\left(d_{1}(x)\right) \\
& \cdot\left(1+C_{2}(N-1)(H(\bar{x})+\epsilon) \frac{K\left(d_{1}(x)\right)}{k\left(d_{1}(x)\right)}\right) \\
& -\frac{2(p-1) C_{1}}{(m+1-p)}\left(K\left(d_{1}(x)\right)\right)^{-\frac{m+p-1}{m+1-p}} k\left(d_{1}(x)\right) \Delta d(x) \\
& \cdot\left(1+C_{2}(N-1)(H(\bar{x})+\epsilon) \frac{K\left(d_{1}(x)\right)}{k\left(d_{1}(x)\right)}\right) \\
& -\frac{4(p-1) C_{1}}{(m+1-p)}\left(K\left(d_{1}(x)\right)\right)^{-\frac{m+p-1}{m+1-p}} \\
& \left.\cdot k\left(d_{1}(x)\right) C_{2}(N-1)(H(\bar{x})+\epsilon) \frac{d}{d t}\left(\frac{K(t)}{k(t)}\right)\right|_{t=d_{1}(x)} \\
& +\left.C_{1} C_{2}(N-1)(H(\bar{x})+\epsilon)\left(K\left(d_{1}(x)\right)\right)^{-\frac{2(p-1)}{m+1-p}} \frac{d^{2}}{d t^{2}}\left(\frac{K(t)}{k(t)}\right)\right|_{t=d_{1}(x)} \\
& +C_{1} C_{2}(N-1)(H(\bar{x})+\epsilon)\left(K\left(d_{1}(x)\right)\right)^{-\frac{2(p-1)}{m+1-p}} \frac{d}{d t} \\
& \left.\left.\cdot\left(\frac{K(t)}{k(t)}\right)\right|_{t=d_{1}(x)} \Delta d(x)\right] \\
& =(p-1)\left(\frac{2(p-1) C_{1}}{m+1-p}\right)^{p-2}\left(K\left(d_{1}(x)\right)\right)^{-\frac{(p-1)(p-2)+m p}{m+1-p}} k^{p}\left(d_{1}(x)\right) \\
& \cdot\left[\frac{2(p-1)(m+p-1) C_{1}}{(m+1-p)^{2}}\right. \\
& +\frac{2(p-1)(m+p-1) C_{1} C_{2}}{(m+1-p)^{2}}(N-1)(H(\bar{x})+\epsilon) \frac{K\left(d_{1}(x)\right)}{k\left(d_{1}(x)\right)} \\
& -\frac{2(p-1) C_{1}}{(m+1-p)}\left(\frac{K\left(d_{1}(x)\right) k^{\prime}\left(d_{1}(x)\right)}{k^{2}\left(d_{1}(x)\right)}-\left(1-C_{k}\right)\right) \\
& -\frac{2(p-1) C_{1}}{(m+1-p)}\left(\frac{K\left(d_{1}(x)\right) k^{\prime}\left(d_{1}(x)\right)}{k^{2}\left(d_{1}(x)\right)}-\left(1-C_{k}\right)\right) \\
& \cdot C_{2}(N-1)(H(\bar{x})+\epsilon) \frac{K\left(d_{1}(x)\right)}{k\left(d_{1}(x)\right)} \\
& -\frac{2(p-1) C_{1}}{(m+1-p)}\left(1-C_{k}\right)\left(1+C_{2}(N-1)(H(\bar{x})+\epsilon) \frac{K\left(d_{1}(x)\right)}{k\left(d_{1}(x)\right)}\right) \\
& -\frac{2(p-1) C_{1}}{(m+1-p)} \frac{K\left(d_{1}(x)\right)}{k\left(d_{1}(x)\right)} \Delta d(x)\left(1+C_{2}(N-1)(H(\bar{x})+\epsilon) \frac{K\left(d_{1}(x)\right)}{k\left(d_{1}(x)\right)}\right) \\
& -\frac{4(p-1) C_{1} C_{2}}{(m+1-p)} \frac{K\left(d_{1}(x)\right)}{k\left(d_{1}(x)\right)}(N-1)(H(\bar{x})+\epsilon)\left(\left.\frac{d}{d t}\left(\frac{K(t)}{k(t)}\right)\right|_{t=d_{1}(x)}\right. \\
& \left.-C_{k}+C_{k}\right)
\end{aligned}
$$




$$
\begin{aligned}
+ & \left.C_{1} C_{2}(N-1)(H(\bar{x})+\epsilon) \frac{K^{2}\left(d_{1}(x)\right)}{k^{2}\left(d_{1}(x)\right)} \frac{d^{2}}{d t^{2}}\left(\frac{K(t)}{k(t)}\right)\right|_{t=d_{1}(x)} \\
& \left.+\left.C_{1} C_{2}(N-1)(H(\bar{x})+\epsilon) \frac{K\left(d_{1}(x)\right)}{k\left(d_{1}(x)\right)} \frac{d}{d t}\left(\frac{K^{2}(t)}{k^{2}(t)}\right)\right|_{t=d_{1}(x)} \Delta d(x)\right] \\
= & (p-1)\left(\frac{2(p-1) C_{1}}{m+1-p}\right)^{p-2}\left(K\left(d_{1}(x)\right)\right)^{-\frac{(p-1)(m+p-1)}{m+1-p}} k^{p-1}\left(d_{1}(x)\right)\left[\frac{k\left(d_{1}(x)\right)}{K\left(d_{1}(x)\right)}\right. \\
& \cdot\left(\frac{2(p-1)(m+p-1) C_{1}}{(m+1-p)^{2}}-\frac{2(p-1) C_{1}}{(m+1-p)}\left(1-C_{k}\right)\right) \\
& +C_{1} C_{2}(N-1)(H(\bar{x})+\epsilon)\left(\frac{2(p-1)(m+p-1)}{(m+1-p)^{2}}-\frac{2(p-1)}{(m+1-p)}\left(1-C_{k}\right)\right. \\
& \left.-\frac{4(p-1) C_{k}}{(m+1-p)}\right)-\frac{2(p-1) C_{1}}{(m+1-p)} \Delta d(x)-\frac{2(p-1) C_{1}}{(m+1-p)}\left(\frac{K\left(d_{1}(x)\right) k^{\prime}\left(d_{1}(x)\right)}{k^{2}\left(d_{1}(x)\right)}\right. \\
& \left.-\left(1-C_{k}\right)\right) \frac{k\left(d_{1}(x)\right)}{K\left(d_{1}(x)\right)}-\frac{2(p-1) C_{1} C_{2}}{(m+1-p)}\left(\frac{K\left(d_{1}(x)\right) k^{\prime}\left(d_{1}(x)\right)}{k^{2}\left(d_{1}(x)\right)}-\left(1-C_{k}\right)\right) \\
& \cdot(N-1)(H(\bar{x})+\epsilon)-\frac{4(p-1) C_{1} C_{2}}{(m+1-p)}(N-1)(H(\bar{x})+\epsilon) \\
& \cdot\left(\left.\frac{d}{d t}\left(\frac{K(t)}{k(t)}\right)\right|_{t=d_{1}(x)}-C_{k}\right) \\
& -\frac{2(p-1) C_{1} C_{2}}{(m+1-p)} \Delta d(x)(N-1)(H(\bar{x})+\epsilon) \frac{K\left(d_{1}(x)\right)}{k\left(d_{1}(x)\right)} \\
+ & \left.\left.C_{1} C_{2}(N-1)(H(\bar{x})+\epsilon) \frac{K\left(d_{1}(x)\right)}{k\left(d_{1}(x)\right)} \frac{d^{2}}{d t^{2}}\left(\frac{K(t)}{k(t)}\right)\right|_{t=d_{1}(x)} \Delta d(x)\right] . \\
+ & \left.C_{1} C_{2}(N-1)(H(\bar{x})+\epsilon) \frac{K\left(d_{1}(x)\right)}{k\left(d_{1}(x)\right)} \frac{d}{d t}\left(\frac{K(t)}{k(t)}\right)\right|_{t=d_{1}(x)}(N)
\end{aligned}
$$

By the value of $C_{1}, C_{2}$ from Theorem 2 and (3.1) we know that

$$
\begin{aligned}
& \frac{2(p-1)(m+p-1) C_{1}}{(m+1-p)^{2}}-\frac{2(p-1) C_{1}\left(1-C_{k}\right)}{(m+1-p)}=\frac{C_{1}^{m}}{p-1}\left(\frac{2(p-1) C_{1}}{m+1-p}\right)^{-(p-2)}, \\
& \frac{2(p-1)(m+p-1) C_{1} C_{2}}{(m+1-p)^{2}}-\frac{2(p-1) C_{1} C_{2}\left(1-C_{k}\right)}{(m+1-p)}-\frac{4(p-1) C_{1} C_{2} C_{k}}{(m+1-p)} \\
& \quad+\frac{2(p-1) C_{1}}{(m+1-p)}=\frac{m C_{1}^{m} C_{2}}{p-1}\left(\frac{2(p-1) C_{1}}{m+1-p}\right)^{-(p-2)},
\end{aligned}
$$

and by Lemmas 1 and 4 we get that there exists $\delta_{\epsilon} \in\left(0, \frac{\delta}{2}\right)$ sufficiently small such that, for $(x, t) \in \Omega_{2 \delta_{\epsilon}} \times\left(0,2 \delta_{\epsilon}\right)$,

$$
\begin{aligned}
-\frac{2(p-1) C_{1}}{(m+1-p)}\left(\frac{K(t) k^{\prime}(t)}{k^{2}(t)}-\left(1-C_{k}\right)\right) \frac{k(t)}{K(t)} & \\
- & \frac{2(p-1) C_{1} C_{2}}{(m+1-p)}\left(\frac{K(t) k^{\prime}(t)}{k^{2}(t)}-\left(1-C_{k}\right)\right) \\
& \cdot(N-1)(H(\bar{x})+\epsilon)-\frac{4(p-1) C_{1} C_{2}}{(m+1-p)}(N-1)(H(\bar{x})+\epsilon)\left(\frac{d}{d t}\left(\frac{K(t)}{k(t)}\right)-C_{k}\right) \\
& -\frac{2(p-1) C_{1} C_{2}}{(m+1-p)} \Delta d(x)(N-1)(H(\bar{x})+\epsilon) \frac{K(t)}{k(t)}
\end{aligned}
$$




$$
\begin{aligned}
& +C_{1} C_{2}(N-1)(H(\bar{x})+\epsilon) \frac{K(t)}{k(t)} \frac{d^{2}}{d t^{2}}\left(\frac{K(t)}{k(t)}\right) \\
& +C_{1} C_{2}(N-1)(H(\bar{x})+\epsilon) \frac{K(t)}{k(t)} \frac{d}{d t}\left(\frac{K(t)}{k(t)}\right) \Delta d(x) \mp \frac{c_{0} C_{1}^{m}}{p-1}\left(\frac{2(p-1) C_{1}}{m+1-p}\right)^{-(p-2)} \\
& \cdot(K(t))^{-\frac{2\left(m_{1}+m\right)(p-1)}{m+1-p}-1} k(t) \hat{L}(\Phi(t))\left(1+C_{2}(N-1)(H(\bar{x})+\epsilon) \frac{K(t)}{k(t)}\right)^{m_{1}} \\
& \leq \frac{C_{1}(N-1)}{m+1-p} \epsilon
\end{aligned}
$$

where $\Phi(t)$ is given in Lemma 4 with $h(t)= \pm C_{2}(N-1)(H(\bar{x})+1) \frac{K(t)}{k(t)}$.

Thus, for $x \in D_{\rho}^{-}$, we have

$$
\begin{aligned}
\Delta_{p} & \bar{u}_{\epsilon}(x)-K^{p-2}\left(d_{1}(x)\right) k^{p}\left(d_{1}(x)\right) f\left(\bar{u}_{\epsilon}(x)\right) \\
\leq & (p-1)\left(\frac{2(p-1) C_{1}}{m+1-p}\right)^{p-2}\left(K\left(d_{1}(x)\right)\right)^{-\frac{(p-1)(m+p-1)}{m+1-p}} \\
& \cdot k^{p-1}\left(d_{1}(x)\right)\left(-\frac{2 C_{1}(N-1)}{m+1-p} \epsilon+\frac{C_{1}(N-1)}{m+1-p} \epsilon\right) \\
\leq & 0
\end{aligned}
$$

i.e., $\bar{u}_{\epsilon}(x)$ is a supersolution of Eq. (1.1) in $D_{\rho}^{-}$.

Similarly, we can show that $\underline{u}_{\epsilon}$ is a subsolution of Eq. (1.1) in $D_{\rho}^{+}$.

By (3.4) and letting $\rho \rightarrow 0$, we have that, for $x \in D_{\rho}^{-} \cap D_{\rho}^{+}$,

$$
\begin{aligned}
& C_{1}(K(d(x)))^{-\frac{2(p-1)}{m+1-p}}\left(1+C_{2}(N-1)(H(\bar{x})+\epsilon) \frac{K(d(x))}{k(d(x))}\right)+C_{1}\left(\delta_{\epsilon}\right) \geq u(x), \\
& C_{1}(K(d(x)))^{-\frac{2(p-1)}{m+1-p}}\left(1+C_{2}(N-1)(H(\bar{x})-\epsilon) \frac{K(d(x))}{k(d(x))}\right)-C_{2}\left(\delta_{\epsilon}\right) \leq u(x) .
\end{aligned}
$$

The proof is complete.

\section{Appendix}

In this appendix, we prove the existence and uniqueness of the solution for problem (1.1).

\section{A.1 The existence of solutions for problem (1.1)}

In the first part, we give the existence of solutions for problem (1.1).

Theorem 3 Let $f$ satisfy $\left(\mathrm{f}_{1}\right)$ and the Keller-Osserman condition (2.7), and b satisfy $\left(\mathrm{b}_{1}\right)$. Then problem (1.1) has at least one solution $u \in W_{\mathrm{loc}}^{1, p}(\bar{\Omega})$ satisfying

$$
u(x) \geq \psi(\bar{v}(x)), \quad \forall x \in \Omega
$$

Furthermore, iff satisfies $\int_{0}^{1} f^{-\frac{1}{p-1}}(s) d s=\infty$, then

$$
u>0, \quad \forall x \in \Omega,
$$


where $\psi$ is the solution of problem (1.4) and $\bar{v} \in W_{0}^{1, p}(\Omega)$ is the unique solution for problem

$$
-\Delta_{p} \bar{v}=b(x), \quad \bar{v}(x)>0, \quad x \in \Omega,\left.\quad \bar{v}\right|_{\partial \Omega}=0 .
$$

Remark 4 By Lemma 2(3), we can see that $f$ satisfies the Keller-Osserman condition under our hypotheses on $f$ in Theorem 1 .

Remark 5 By the similar argument in [46], we show that $\left(f_{1}\right)$ and the Keller-Osserman condition imply $\left(\mathrm{f}_{2}\right)$. Indeed, if we can prove that there exist two positive numbers $\rho$ and $M$ such that

$$
\frac{f^{p^{\prime}-1}(s)}{s} \geq \rho^{p^{\prime}} \quad \text { for } s \geq M,
$$

then it will be done since

$$
F(s)=\int_{0}^{s} f(t) d t \leq s f(s) \leq \frac{f^{p^{\prime}}(s)}{\rho^{p^{\prime}}} \quad \text { for } s \geq M,
$$

which, in turn, yields $[F(s)]^{-\frac{1}{p}} \geq \frac{\rho^{\frac{1}{p-1}}}{f^{\frac{1}{p-1}(s)}}$, so that the Keller-Osserman condition implies (A.4). Then, we will prove (A.4) by contradiction. Assume that there exists an increasing sequence $s_{j}$ of real numbers such that $\lim _{j \rightarrow \infty} s_{j}=\infty$ and $\frac{f\left(s_{j}\right)}{s_{j}}<\frac{1}{j}$ for all $j$. Since $f$ is increasing, we have $f(s) \leq f\left(s_{j}\right)$ for all $s \in\left[0, s_{j}\right]$, which, in turn, produces $F(s) \leq s f(s) \leq s f\left(s_{j}\right) \rightarrow \infty$ for $s \in\left[0, s_{j}\right]$. Hence,

$$
\begin{aligned}
\int_{s_{1}}^{s_{j}}[F(s)]^{-\frac{1}{p}} d s & \geq \int_{s_{1}}^{s_{j}}[F(s)]^{-\frac{1}{p}} d s \geq\left[\frac{j}{s_{j}}\right]^{\frac{1}{p^{\prime}}} \int_{s_{1}}^{s_{j}} s^{-\frac{1}{p}} d s \\
& =\frac{p}{p-1} j^{\frac{1}{p^{\prime}}}\left(1-\left(\frac{s_{1}}{s_{j}}\right)\right) \rightarrow \infty
\end{aligned}
$$

as $j \rightarrow \infty$, which contradicts the Keller-Osserman condition (2.7). Thus, (A.4) must be true, and then $\left(f_{2}\right)$ holds.

Proof Let

$$
v=\phi(u)=\int_{u}^{\infty} f^{-\frac{1}{p-1}}(v) d v, \quad u>0 .
$$

We see that problem (1.1) is equivalent to the following problem:

$$
-\Delta_{p} v+g(v)|\nabla v|^{p}=b(x), \quad v>0, x \in \Omega,\left.\quad \bar{v}\right|_{\partial \Omega}=0,
$$

where $g(v)=f^{\prime}(\psi(v))$, and $\phi$ is also the inverse function of $\psi$.

Now let $v \in W_{\text {loc }}^{1, p}(\Omega) \cap L_{\text {loc }}^{\infty}(\Omega)$ be any solution of problem (A.6). We claim that

$$
v(x) \leq \bar{v}(x), \quad \forall x \in \Omega
$$


Indeed, assume on the contrary that $\{x \in \Omega: v(x)>\bar{v}(x)\} \neq \emptyset$. Then, on its arbitrary connected component $D$, we have $-\Delta_{p}(v-\bar{v})(x) \leq 0, x \in D$, since $g(v) \geq 0$. It follows by $\left.(v-\bar{v})\right|_{\partial \Omega}=0$ and the maximum principle that $v(x) \leq \bar{v}(x)$ for all $x \in D$. This is a contradiction. Thus, (A.7) holds, i.e., any weak solution $u$ of problem (1.1) satisfies (A.1). Moreover, by the definition of $\psi$ and the condition $\int_{0}^{1} \frac{d s}{f(s)}=\infty$ we see that $\psi(\bar{v}(x))>0, \forall x \in \Omega$, and (A.2) holds. Next, we consider the perturbed problem

$$
\Delta_{p} u=b(x) f(u), \quad x \in \Omega,\left.\quad u\right|_{\partial \Omega}=m \in N .
$$

By $\left(\mathrm{b}_{1}\right)$ and $\left(\mathrm{f}_{1}\right)$ we see that $\bar{u}_{m}=m$ is a supersolution of problem (A.8). To construct a subsolution $\underline{u}_{1}$ of problem (A.8), we let $\bar{v}_{1} \in C^{2+\alpha}(\bar{\Omega})$ be the unique solution of the problem

$$
-\Delta_{p} \bar{v}_{1}=b(x), \quad \bar{v}(x)>0, \quad x \in \Omega,\left.\quad \bar{v}_{1}\right|_{\partial \Omega}=\int_{1}^{\infty} f^{-\frac{1}{p-1}}(s) d s,
$$

and $\bar{u}_{1}=\psi\left(\bar{v}_{1}\right)$. Then we see that $\left.\underline{u}_{1}\right|_{\partial \Omega}=1 \leq m$ and

$$
-\Delta_{p} \bar{\nu}_{1}=\frac{\Delta_{p} \underline{u}_{1}}{f\left(\underline{u}_{1}\right)}-\frac{f^{\prime}\left(\underline{u}_{1}\right)}{f^{2}\left(\underline{u}_{1}\right)}\left|\nabla \underline{u}_{1}\right|^{p}=b(x), \quad x \in \Omega,
$$

which yields

$$
\Delta_{p} \underline{u}_{1} \geq b(x) f\left(\underline{u}_{1}\right), \quad x \in \Omega,
$$

i.e., $\underline{u}_{1}$ is a subsolution of problem (A.8). Moreover, $\underline{u}_{1} \leq 1 \leq m, x \in \Omega$, due to the maximum principle. Thus, problem (A.8) has one solution $u_{m} \in W_{0}^{1, p}$ in the order interval $\left[u_{1}, m\right]$, and the maximum principle again yields that the map $m \rightarrow u_{m}$ is increasing. On the other hand, the classical Keller-Osserman condition guarantees that the problem

$$
\Delta_{p} u=b_{0} f(u), \quad x \in \Omega_{0},\left.\quad u\right|_{\partial \Omega_{0}}=\infty,
$$

has one solution $u_{\Omega_{0}} \in W_{\text {loc }}^{1, p}(\Omega)$ for each $\Omega_{0} \subset \subset \Omega$, where $b_{0}=\min _{x \in \bar{\Omega}_{0}} b(x)$. By the maximum principle we have $u_{m} \leq u_{\Omega_{0}}(x), x \in \Omega_{0}$, and $u(x):=\lim _{m \rightarrow \infty} u_{m}(x)$ exists for $x \in \Omega_{0}$. Thus, $u$ is the desired solution for problem (1.1) by the standard bootstrap argument, the arbitrariness of $\Omega_{0}$, and (A.1).

\section{A.2 The uniqueness of the solution for problem (1.1)}

In the second part, we prove the uniqueness of the solution for problem (1.1). The method is similar to the idea in $[40,58]$.

Theorem 4 Under the hypotheses in Theorem 1, problem (1.1) admits a unique solution.

Proof Since $\frac{f(s)}{s^{q}}$ is increasing in $\left[S_{0}, \infty\right)$ for some $q>p-1$ and $S_{0}$ large enough, by Lemma 2(2) we have

$$
\frac{f(s)}{s} \text { is also increasing in }\left[S_{0}, \infty\right) \text {. }
$$


Let $u_{0}$ be the minimal solution for problem (1.3), and $u$ be another solution for problem (1.1). We prove that $u=u_{0}$ in $\Omega$. In fact, by the maximum principle we have

$$
u_{0} \leq u \quad \text { in } \Omega
$$

Moreover, by the asymptotic behavior (1.3) we deduce that

$$
\lim _{d(x) \rightarrow 0} \frac{u_{0}(x)}{u(x)}=1
$$

For any $\epsilon>0$, setting $w=(1+\epsilon) u_{0}$, we have

$$
\lim _{d(x) \rightarrow 0}(w(x)-u(x))=\lim _{d(x) \rightarrow 0}\left(\frac{(1+\epsilon) u_{0}(s)}{u(s)}-1\right)=+\infty
$$

Now, for small $\epsilon>0$, we define the open set

$$
D_{\epsilon}=\{x \in \Omega: w(x)<u(x)\} .
$$

We may assume that $D_{\epsilon}$ is nonempty for $\epsilon$ small enough; otherwise, there is nothing to prove. Indeed, notice that $D_{\epsilon}$ increases as $\epsilon \rightarrow 0$. Moreover, we may also assume that $D_{\epsilon} \rightarrow \Omega$ as $\epsilon \rightarrow 0$; if there exists $x_{0} \in \Omega$ and a sequence $\epsilon_{n} \rightarrow 0$ such that $x_{0} \in D_{\epsilon_{n}}$ for all $n$, then we have $\left(1+\epsilon_{n}\right) u_{0}\left(x_{0}\right) \geq u\left(x_{0}\right)$. Then the strong maximum principle yields $u \equiv u_{0}$ in $\Omega$. Finally, we have $D_{\epsilon} \subset \Omega$ by (A.13).

Next, we choose $\eta>0$ such that $u_{0} \geq S_{0}$ in $\Omega_{\eta}$ and define $D_{\epsilon, \eta}=D_{\epsilon} \cap D_{\eta}$. Notice that $D_{\epsilon, \eta}$ is a nonempty open set for small $\epsilon$. Moreover, by (A.11) we have

$$
\Delta_{p} w=(1+\epsilon) b(x) f\left(u_{0}\right) \leq b(x) f(w), \quad x \in D_{\epsilon, \eta} .
$$

It follows by $\left(\mathrm{f}_{1}\right)$ that

$$
\Delta_{p}(u-w) \geq b(x)(f(u)-f(w)) \geq 0, \quad x \in D_{\epsilon, \eta} .
$$

Thus, by the maximum principle we obtain

$$
u(x)-w(x) \leq \max _{\partial D_{\epsilon, \eta}}(u-w), \quad x \in D_{\epsilon, \eta} .
$$

Since $\partial D_{\epsilon, \eta}=\left(\partial D_{\epsilon} \cap D_{\eta}\right) \cup\left(D_{\epsilon} \cap \partial D_{\eta}\right), D_{\epsilon} \cap \partial \Omega=\emptyset$, and $\left.(u-w)\right|_{\partial D_{\epsilon}}=0$, we see that the maximum of $u-w$ is achieved on $D_{\epsilon} \cap \partial D_{\eta}=\left(D_{\epsilon} \cap x: d(x)=\eta\right)$. Hence,

$$
u(x)-w(x) \leq \max _{\left(D_{\epsilon} \cap x: d(x)=\eta\right)}(u-w), \quad x \in D_{\epsilon, \eta}
$$

Letting $\epsilon \rightarrow 0$ in (A.19), we obtain

$$
u-u_{0} \leq \max _{d(x)=\eta}\left(u-u_{0}\right):=\theta \quad \text { in } \Omega_{\eta}
$$


On the other hand, by (A.12) and $\left(f_{1}\right)$ we have

$$
\Delta_{p}\left(u-u_{0}\right)=b(x)\left(f(u)-f\left(u_{0}\right)\right) \geq 0, \quad x \in \Omega^{\eta}=x \in \Omega: d(x)>\eta .
$$

The maximum principle implies that $u-u_{0} \leq \theta$ in $\Omega^{\eta}$, and hence $u-u_{0} \leq \theta$ in the whole $\Omega$. Then the strong maximum principle gives $u-u_{0} \equiv \theta$. We obtain that $f(u)=f(u+\theta)$ in $\Omega$, which can only hold if $\theta=0$. Thus, $u=u_{0}$, which shows the uniqueness.

\section{Competing interests}

The authors declare that they have no competing interests.

\section{Authors' contributions}

All authors contributed equally to the manuscript and read and approved the final manuscript.

\section{Author details}

${ }^{1}$ Department of Fundamental Courses, Qingdao College, Qingdao Technological University, Qingdao, 266106, P.R. China.

${ }^{2}$ School of Mathematical Sciences, Ocean University of China, Songling Road 238, Qingdao, 266100, P.R. China.

\section{Acknowledgements}

This work is supported by the Natural Science Foundation of Shandong Province of China (ZR2012AM018) and the Fundamental Research Funds for the Central Universities (No. 201362032). The authors would like to deeply thank all the reviewers for their insightful and constructive comments.

Received: 29 July 2015 Accepted: 26 November 2015 Published online: 10 December 2015

\section{References}

1. Kawohl, B: On a family of torsional creep problems. J. Reine Angew. Math. 410, 1-22 (1990)

2. Showalter, RE, Walkington, NJ: Diffusion of fluid in a fissured medium with microstructure. J. Math. Anal. 22, 1702-1722 (1991)

3. Plissier, MC, Reynaud, ML: Étude d'un modèle mathématique d'écoulement de glacier. C. R. Acad. Sci. Paris, Ser. I Math. 279, 531-534 (1974)

4. Bieberbach, L: $\Delta u=e^{u}$ und die automorphen Funktionen. Math. Ann. 77, 173-212 (1916)

5. Rademacher, $\mathrm{H}$ : Einige besondere probleme partieller differentialgleichungen. In: Die Differential- und Integralgleichungen der Mechanik und Physik, 2nd edn., vol. I, pp. 838-845. Rosenberg, New York (1943)

6. Keller, JB: On solutions of $\Delta u=f(u)$. Commun. Pure Appl. Math. 10,503-510 (1957)

7. Zhang, ZJ: A remark on the existence of explosive solutions for a class of semilinear elliptic equations. Nonlinear Anal. TMA 41, 143-148 (2000)

8. Tao, SP, Zhang, ZJ: On the existence of explosive solutions for semilinear elliptic problems. Nonlinear Anal. TMA 48, 1043-1050 (2002)

9. Huang, SB, Tian, QY, Zhang, SZ, Xi, JH: A second order estimate for blow-up solutions of elliptic equations. Nonlinear Anal. TMA 74, 2342-2350 (2011)

10. Huang, SB, Tian, QY, Zhang, SZ, Xi, JH, Fan, ZG: The exact blow-up rates of large solutions for semilinear elliptic equations. Nonlinear Anal. TMA 73, 3489-3501 (2010)

11. Bandle, C, Giarrusso, E: Boundary blow-up for semilinear elliptic equations with nonlinear gradient terms. Adv. Differ. Equ. 1, 133-150 (1996)

12. Lair, AV: A necessary and sufficient condition for existence of large solutions to semilinear elliptic equations. J. Math Anal. Appl. 240, 205-218 (1999)

13. $\mathrm{Du}, \mathrm{Y}, \mathrm{Ma}, \mathrm{L}$ : Logistic type equations on $R^{N}$ by a squeezing method involving boundary blow-up solutions. J. Lond. Math. Soc. 64, 107-124 (2001)

14. Arrieta, JM, Rodriguez-Bernal, A: Localization near the boundary of blow-up for reaction-diffusion equations with nonlinear boundary conditions. Commun. Partial Differ. Equ. 29, 1127-1148 (2004)

15. Du, Y, Li, S: Positive solutions with prescribed patterns in some simple semilinear equations. Differ. Integral Equ. 15, 805-822 (2002)

16. Du, Y, Yamada, Y: On the long-time limit of positive solutions to the degenerate logistic equation. Discrete Contin. Dyn. Syst., Ser. A 25, 123-132 (2009)

17. Cirstea, F-C, Rădulescu, V: Uniqueness of the blow-up boundary solution of logistic equations with absorbtion. C. R. Acad. Sci. Paris, Ser. I 335, 447-452 (2002)

18. Cirstea, F-C, Rădulescu, V: Asymptotics for the blow-up boundary solution of the logistic equation with absorbtion. C. R. Acad. Sci. Paris, Ser. I 336, 231-236 (2003)

19. Cirstea, F-C, Rădulescu, V: Nonlinear problems with boundary blow-up: a Karamata regular variation theory approach. Asymptot. Anal. 46, 275-298 (2006)

20. Cirstea, F-C, Rădulescu, V: Boundary blow-up in nonlinear elliptic equations of Bieberbach-Rademacher type. Trans. Am. Math. Soc. 359, 3275-3286 (2007)

21. Garcia-Melián, J: Boundary behavior of large solutions to elliptic equations with singular weights. Nonlinear Anal. TMA 67, 818-826 (2007)

22. Lazer, AC, McKenna, PJ: Asymptotic behavior of solutions of boundary blowup problems. Differ. Integral Equ. 7 , 1001-1019 (1994) 
23. Du, Y, Huang, Q: Blow-up solutions for a class of semilinear elliptic and parabolic equations. SIAM J. Math. Anal. 31, 1-18 (1999)

24. Cano-Casanova, S, López-Gómez, J: Blow-up rates of radially symmetric large solutions. J. Math. Anal. Appl. 352(1), 166-174 (2009)

25. López-Gómez, J: Optimal uniqueness theorems and exact blow-up rates of large solutions. J. Differ. Equ. 224, 385-439 (2006)

26. López-Gómez, J: Uniqueness of radially symmetric large solutions (Proc. 6th AIMS Int. Conf., suppl.). Discrete Contin. Dyn. Syst. 2007, 677-686 (2007)

27. Loewner, C, Nirenberg, L: Partial differential equations invariant under conformal or projective transformations. In: Contributions to Analysis, pp. 245-272. Academic Press, Orlando (1974)

28. Bandle, C, Marcus, M: Large solutions of semilinear elliptic equations: existence, uniqueness and asymptotic behavior. J. Anal. Math. 58, 9-24 (1992)

29. Bandle, C, Marcus, M: On second-order effects in the boundary behavior of large solutions of semilinear elliptic problems. Differ. Integral Equ. 11, 23-34 (1998)

30. Bandle, C: Asymptotic behavior of large solutions of quasilinear elliptic problems. Z. Angew. Math. Phys. 54, 731-738 (2003)

31. Bandle, C, Marcus, M: Dependence of blowup rate of large solutions of semilinear elliptic equations, on the curvature of the boundary. Complex Var. Theory Appl. 49, 555-570 (2004)

32. Anedda, C, Porru, G: Boundary behavior for solutions of boundary blow-up problems in a borderline case. J. Math. Anal. Appl. 352, 35-47 (2009)

33. Garcia-Meliaan, J, Letelier-Albornoz, R, Sabinade Lis, J: Uniqueness and asymptotic behaviour for solutions of semilinear problems with boundary blow-up. Proc. Am. Math. Soc. 129(12), 3593-3602 (2001)

34. del Pino, M, Letelier, R: The influence of domain geometry in boundary blow-up elliptic problems. Nonlinear Anal. TMA 48, 897-904 (2002)

35. Cirstea, F-C: Elliptic equations with competing rapidly varying nonlinearities and boundary blow-up. Adv. Differ. Equ. $12,995-1030$ (2007)

36. Diaz, G, Letelier, R: Explosive solutions of quasilinear elliptic equations: existence and uniqueness. Nonlinear Anal. TMA 20, 97-125 (1993)

37. Zhang, ZJ: The asymptotic behaviour of solutions with blow-up at the boundary for semilinear elliptic problems. J. Math. Anal. Appl. 308, 532-540 (2005)

38. Zhang, ZJ: The second expansion of large solutions for semilinear elliptic equations. Nonlinear Anal. TMA 74, 3445-3457 (2011)

39. Zhang, ZJ: The second expansion of the solution for a singular elliptic boundary value problem. J. Math. Anal. Appl. 381, 922-934 (2011)

40. Zhang, ZJ, Ma, YJ, Mi, L, Li, XH: Blow-up rates of large solutions for elliptic equations. J. Differ. Equ. 249, 180-199 (2010)

41. Lu, QS, Yang, ZD, Twizell, EH: Existence of entire explosive positive solutions of quasi-linear elliptic equations. Appl. Math. Comput. 148, 359-372 (2004)

42. Yang, ZD, Xu, B, Wu, MZ: Existence of positive boundary blow-up solutions for quasilinear elliptic equations via sub and supersolutions. Appl. Math. Comput. 188, 492-498 (2007)

43. Yang, ZD: Existence of explosive positive solutions of quasilinear elliptic equations. Appl. Math. Comput. 177, 581-588 (2006)

44. McKenna, PJ, Reichel, TW, Walter, W: Symmetry and multiplicity for nonlinear elliptic differential equations with boundary blow up. Nonlinear Anal. TMA 28, 1213-1225 (1997)

45. Serrin, J: Entire solutions of quasilinear elliptic equations. J. Math. Anal. Appl. 352, 3-14 (2009)

46. Huang, SB, Tian, QY: Asymptotic behavior of large solution for boundary blowup problems with non-linear gradient terms. Appl. Math. Comput. 215, 3091-3097 (2009)

47. Mohammed, A: Existence and asymptotic behavior of blow-up solutions to weighted quasilinear equations. J. Math. Anal. Appl. 298, 621-637 (2004)

48. Mohammed, A: Boundary asymptotic and uniqueness of solutions to the $p$-Laplacian with infinite boundary values. J. Math. Anal. Appl. 325, 480-489 (2007)

49. Guo, ZM, Webb, JRL: Structure of boundary blow-up solutions for quasi-linear elliptic problems II: small and intermediate solutions. J. Differ. Equ. 211, 187-217 (2005)

50. Covei, DP: Large and entire large solution for a quasilinear problem. Nonlinear Anal. TMA 70, 1738-1745 (2009)

51. Melian, J, Rossi, JD, Sabina, J: Large solutions to the $p$-Laplacian for large $p$. Calc. Var. Partial Differ. Equ. 31, 187-204 (2008)

52. Zhang, Q, Liu, X, Qiu, Z: On the boundary blow-up solutions of $p(x)$-Laplacian equations with singular coefficient. Nonlinear Anal. TMA 70, 4053-4070 (2009)

53. Zhang, Q: Existence and asymptotic behavior of blow-up solutions to a class of $p(x)$-Laplacian problems. J. Math. Anal. Appl. 329, 472-482 (2007)

54. Du, Y, Guo, ZM: Boundary blow up solutions and their applications in quasilinear elliptic equations. J. Anal. Math. 89 277-302 (2003)

55. Marić, V: Regular Variation and Differential Equations. Lecture Notes in Math., vol. 1726. Springer, Berlin (2000)

56. Resnick, SI: Extreme Values, Regular Variation, and Point Processes. Springer, New York (1987)

57. Seneta, R: Regular Varying Functions. Lecture Notes in Math., vol. 508. Springer, Berlin (1976)

58. Garcia-Meliaan, J: Uniqueness of positive solutions for a boundary blow-up problem. J. Math. Anal. Appl. 360(2), 530-536 (2009) 